\title{
Crowning during Childbirth
}

National Cancer Institute

\section{Source}

National Cancer Institute. Crowning during Childbirth. NCI Thesaurus. Code C92763.

The characteristic sign of impending birth during the second stage of labor. The presenting part of the head of fetus is visible and firmly rimmed by the maternal vaginal wall. 